\title{
Grupo operativo como ferramenta para o cuidado em saúde mental
}

\author{
Operative group as a tool for metal health care \\ Grupo terapéutico: una tecnologia del cuidado en salud mental
}

Recebido: 25/02/2021 | Revisado: 09/03/2021 | Aceito: 15/03/2021 | Publicado: 21/03/2021

Maria Luana Mota Sousa
ORCID: https://orcid.org/0000-0002-6385-2708
E-mail: luanamota344@ @gmail.com
Universidade da Integração Internacional da Lusofonia Afro-Brasila, Brasi
Hévila Ferreira Gomes Medeiros Braga
ORCID: https://orcid.org/0000-0003-4188-2882
E-mail: hevila.medeiros.hm@ @mail.com
Albertina Antonielly Sydney de Sousa
ORCID: https://orcid.org/0000-0002-1625-1889
Escola de Saúde Pública do Ceará, Brasil
E-mail: albertinasydney@gmail.com
Universidade da Integração Internacional da Lusofonia Afro-Brasileira, Brasil
Carolina Maria de Lima Carvalho
ORCID: https://orcid.org/0000-0002-5173-5360
E-mail: carolinacarvalho@ unilab.edu.br
Sheila Mirelly Goncalves Arrais
ORCID: https://orcid.org/0000-0002-6004-2531
Secretaria Municipal de Saúde de Fortaleza, Brasil
E-mail: sheilamgarrais@ gmail.com
Eysler Gonçalves Maia Brasil
Universidade da Integração Internacional da Lusofonia Aro-Brasileir, Brasi
ORCID: https://orcid.org/0000-0002-4126-2256
E-mail: eyslerbrasil@unilab.edu.br

\section{Resumo}

Objetivo: relatar a experiência por acadêmicos do curso de enfermagem sobre a realização de um grupo operativo com o tema "motivação para o tratamento" para as pessoas com necessidades decorrentes do uso de álcool, crack e outras drogas. Metodologia: Estudo descritivo, tipo relato de experiência, realizado durante as práticas da disciplina Processo de cuidar na saúde mental da Universidade da Integração Internacional da Lusofonia Afro-Brasileira, durante o mês de julho/2019. Participaram da atividade grupal 19 usuários de uma Unidade de Acolhimento, na cidade de Fortaleza-CE. Resultados: a ação foi realizada em três momentos, sendo eles "aproximação com o grupo, apresentação da temática e realização da tarefa, e o encerramento". Os participantes demonstraram que esse tipo de ação é efetiva, para compreender melhor as barreiras e dificuldades enfrentadas por eles e melhorar as intervenções, ademais, abordar a temática em questão se torna uma prioridade para a superação na fase de abstinência da substância. Considerações finais: observou-se a existência de influência positiva do grupo operativo para os integrantes da unidade que relataram ser uma experiência necessária para seu tratamento e para os discentes foi possível conhecer melhor na prática a dinâmica para realização dos grupos.

Palavras-chave: Usuário de drogas; Saúde mental; Enfermagem.

\begin{abstract}
Objective: to report the experience of an operative group on "motivation for treatment" for people with needs derived from the consumption of alcohol, crack and other drugs. Method: Descriptive study, as a experience report, carried out during the practices of the discipline. Care process in mental health from the University of the International Integration of Lusofonia Afro-Brazilian, during the month of July / 2019. 19 users of a host unit, in the city of Fortaleza-CE, will attend the group activity. The action was executed in three moments: "the approach with the group, theme's presentation and performance of the subject, and the closure." Results: They prove that this type of action is effective to understand better the obstacles and difficulties that users face, to improve interventions, in addition, face the issue becomes a basic need for them to overcome the substance's phase withdrawal . Conclusion: the existence of a positive influence of the operative group was confirmed for the members of the unit who reported it to be a fundamental experience for their treatment and for the students it was possible to understand in practice better the dynamics for conducting the groups.
\end{abstract}

Keywords: Drug users; Mental health; Nursing. 


\begin{abstract}
Resumen
Objetivo: Relatar la experiencia de un grupo operativo sobre "motivación para el tratamiento" para las personas con necesidades derivadas del consumo de alcohol, crack y otras drogas. Método: Estudio descriptivo, tipo relato de experiencia, realizado durante las prácticas de la disciplina Proceso de cuidados en la salud mental de la Universidad de la Integración Internacional de Lusofonia Afro-Brasileira, durante el mes de julio/2019. Participarán de la actividad grupal 19 usuarios de una Unidad de Acogida, en la ciudad de Fortaleza-CE. La acción fue realizada en tres momentos, siendo ellos "la aproximación con el grupo, presentación de la temática y realización de la actividad, y el cierre". Resultados: Demostraron que ese tipo de acción es efectivo para comprender mejor las barreras y dificultades que los usuarios enfrentan, para mejorar las intervenciones, además, abordar la temática en cuestión se torna una necesidad fundamental para que ellos superen la fase de abstinencia de la sustancia. Conclusión: se confirmó la existencia de influencia positiva del grupo operativo para los integrantes de la unidad que relataron ser una experiencia necesaria para su tratamiento y para los estudiantes fue posible conocer mejor en la práctica la dinámica para la realización de los grupos.
\end{abstract}

Palabras clave: Consumidor de drogas; Salud mental; Enfermería.

\title{
1. Introdução
}

No decorrer dos últimos anos o número de pacientes dependentes de álcool e outras drogas têm crescido de forma significante em diversos países e, paralelamente, observa-se a necessidade de intervenções com tratamentos específicos por meio de profissionais qualificados. Esses pacientes sofrem por fazer uso abusivo de substâncias psicoativas que causam alterações no sistema nervoso central, e podem levar à dependência (Soares, Esswein \& Benetti, 2017). Tais substâncias ocasionam graves impactos na saúde física e mental dos dependentes químicos a partir de consequências nocivas na condição física, no autocuidado, no pensamento, na cognição e no comportamento (Silva et al, 2016).

No Brasil, os pacientes que apresentam transtornos mentais e que são atendidos no âmbito do Sistema Único de Saúde (SUS), recebem atendimento na Rede de Atenção Psicossocial (RAPS) que visa articular ações e serviços de saúde em diferentes níveis de complexidade, tais como nos CAPS (Centro de Atenção Psicossocial) em suas diferentes modalidades, no Serviço Residencial Terapêutico (SRT), nas Unidades de Acolhimento (adulto e infanto-juvenil), nas comunidades terapêuticas, entre outros (Brasil, 2017).

Os modelos de tratamento necessitam de intervenções que incluam diversas estratégias de abordagem do problema e contribuam para facilitar o processo de integração dos profissionais com os usuários, levando em consideração, principalmente, dois agravantes responsáveis por recaídas frequentes: a baixa adesão e a falta de motivação para o tratamento. Dentre estas abordagens, existe o grupo operativo, que constitui um dos principais recursos terapêuticos nos mais diferentes contextos de assistência à saúde, e decorre, em grande parte, das condições criadas a partir da reforma psiquiátrica, tendo como foco a ressocialização do indivíduo em sofrimento psíquico (Inoue et al, 2019).

Em vista disso, na concepção de Pichon-Rivière, médico psiquiatra que teorizou essa técnica grupal, o grupo operativo apresenta-se como instrumento de transformação da realidade, no qual seus integrantes passam a estabelecer relações grupais, na medida que começam a partilhar objetivos em comuns que contribuem para que tenham uma participação criativa e crítica, além de poder perceber como interagem e se vinculam, buscando a transformação de si, dos outros e do contexto em que estão inseridos (Bastos \& Bastos, 2010).

A mudança é um dos principais objetivos do grupo operativo. Ademais, vale ressaltar que essa estratégia também é essencial para dar suporte, fortalecer, ampliar o conhecimento dos participantes e auxiliar no enfrentamento de situações desafiadoras e das lutas diárias, além de, realizar as orientações acerca do autocuidado. Assim, o grupo não é um simples ajuntamento de pessoas, mas, um sistema identificável, composto de três ou mais indivíduos que se engajam em tarefas a fim de alcançar um objetivo comum (Moraes, Lopes \& Braga, 2006).

O trabalho com essa modalidade grupal tem sido utilizado por profissionais de saúde, tendo em vista a capacidade operativa e educativa do grupo em promover o acolhimento, ressignificação das experiências e a aprendizagem construída com 
a interação dos participantes (Oliveira et al, 2016). Nessa perspectiva, pôr a enfermagem ser a categoria profissional que comumente realiza atividades grupais nos diversos cenários de assistência em saúde, o enfermeiro se destaca como um dos principais atores que integra a equipe interdisciplinar na abordagem à pessoa dependente de substâncias psicoativas, exigindo desse profissional condições técnicas que viabilizam trabalhar com as limitações do cliente e com os determinantes do processo saúde doença mental, sendo que estas devem ser identificadas e trabalhadas com a ajuda de uma equipe multidisciplinar (Pinheiro et al, 2019).

Diante disso, um dos principais desafios para o ensino de enfermagem em saúde mental é instrumentalizar os alunos para agirem em situações de incertezas por meio de práticas profissionais sistematizadas, criativas e autônomo dialógicas que proporcione o desenvolvimento de um atendimento integral e interdisciplinar (Tavares \& Mesquita, 2019).

O grupo operativo favorece aos participantes o reconhecimento de suas necessidades e contribui no alcance da mudança. Sendo assim, para as pessoas que se encontram em processo de reabilitação decorrente do uso de substâncias psicoativas, o grupo operativo visando à motivação para o tratamento dessas pessoas, é uma estratégia essencial, a temática abordada durante o grupo mostra que muitos tem a iniciativa de dar o primeiro passo, mas não encontram apoio da família e amigos, e poucas vezes até mesmo na unidade de saúde, sendo o tema primordial que incentivam eles a continuarem no processo de recuperação e aprender a conviver sem o uso da substância.

Desta forma, "a motivação para o tratamento é considerada um componente importante no tratamento dos toxicodependentes, quer como o elemento que desencadeia o tratamento, quer enquanto foco do próprio processo terapêutico" (Janeiro, 2017). Frente ao exposto, o objetivo deste trabalho foi relatar a experiência por acadêmicos do curso de enfermagem sobre a realização de um grupo operativo com o tema "motivação para o tratamento" para as pessoas com necessidades decorrentes do uso de álcool, crack e outras drogas.

\section{Metodologia}

Trata-se de um estudo descritivo, com abordagem qualitativa, do tipo relato de experiência. $\mathrm{O}$ cenário da vivência foi uma Unidade de Acolhimento, em Fortaleza-CE que oferece acolhimento residencial transitório às pessoas de ambos os sexos com necessidades decorrentes do uso de álcool, crack e outras drogas. Na unidade, os usuários são acompanhados por uma equipe multiprofissional, formada por enfermeiro, psicólogo, assistente social, auxiliar enfermagem, educador físico e educadores sociais. A instituição funciona no período de 24 horas, possui 30 vagas para tratamento de homens e mulheres.

A coleta de dados deu-se a partir de um roteiro de planejamento para realização do grupo operativo. A atividade foi realizada como parte dos requisitos finais de avaliação do período de estágio da disciplina de Processo de Cuidar na Saúde Mental, nas segundas e terças do mês de julho de 2019.

No período de realização do presente estudo, encontravam-se na Unidade de Acolhimento 20 usuários, dos quais 19 participaram da atividade grupal proposta. Ressalta-se que a única pessoa que não participou foi devido à condição de estar acamado. Para a realização do grupo, os usuários foram consultados previamente pelos acadêmicos acerca da temática que eles gostariam que fosse abordada. Desse modo, foi mencionado como de preferência dialogar sobre "motivação para o tratamento". O grupo foi planejado para ser realizado em três momentos e definido o tempo máximo de 40 minutos, para não tornar a atividade cansativa para os usuários da unidade.

De início, houve uma apresentação do grupo, foi aplicada uma dinâmica de "quebra-gelo" para que os participantes pudessem interagir entre si e com os acadêmicos, refletindo sobre o seu estado atual de vida e planejamento para o futuro. A dinâmica consistiu na entrega de papéis para cada participante para o desenho ou escrita de texto que representasse a sua maior motivação para continuar no tratamento e suas metas. Este primeiro momento foi concluído com um poema com a temática "sonhos e desafios", cuja autora é Ivone Boechat. 
No segundo momento, foi apresentada a temática a ser trabalhada relatando sobre os estágios de mudança. Depois, eles foram convidados a participarem de uma tarefa que consistia em utilizar o instrumento feito pelos acadêmicos, cuja meta era relatar em qual estágio eles se encontravam.

No último momento, foi utilizado a música "É preciso saber viver", e entregue balões que possuíam frases de motivação, para tornar a atividade mais descontraída e fazer um momento diferente. Por fim, foi colocado um vídeo do poeta nordestino Bráulio Bessa sobre "não desistir dos seus sonhos".

Os participantes foram convidados uma semana anterior por meio do contato verbal, reforçando o tema selecionado. Ademais, o espaço planejado para a atividade foi a biblioteca, por ser um local reservado, fechado, com capacidade de acomodar todos que participaram. Os materiais utilizados foram o projetor, a caixa de som, papéis em branco, lápis de cor e o instrumento construído para a terceira parte da atividade.

Os dados acerca da vivência foram registrados no diário de campo dos acadêmicos e foram analisados com base na literatura pertinente ao tema. Por se tratar de relato de experiência, salienta-se a dispensa da necessidade de aprovação por Comitê de Ética em Pesquisa. Porém, frisa-se o respeito deste estudo aos preceitos da Resolução 466 de 2012 que trata da pesquisa com seres humanos, garantindo-se o sigilo das informações e anonimato dos participantes.

\section{Resultados e Discussão}

A realização do grupo operativo teve duração de uma hora e meia, superando o que se esperava no planejamento da atividade, e contou com a participação de 19 usuários da Unidade de Acolhimento. A descrição da vivência foi organizada de acordo com os momentos da atividade e se encontra descrita a seguir.

\subsection{Primeiro momento - Aproximação com o grupo}

Inicialmente, os participantes foram bem interativos e se apresentaram de maneira cordial, descontraída. A maioria relatou e as principais metas para o futuro se referem a deixar o vício e não ter recaídas, voltar a conviver com a família e ter um trabalho fixo. Na dinâmica, foi orientado a importância de ter um planejamento para o futuro, pois servirá de base para a motivação e a superação dos problemas que possam aparecer no decorrer do tratamento. Durante a leitura do poema "sonhos e desafios" eles reagiram de forma positiva e relataram a dificuldade que é estar longe dos familiares e amigos.

\section{2 Segundo momento - Apresentação da temática e realização da tarefa}

A mudança comportamental é um processo no qual o dependente químico exerce papel central para a busca de mudança e de hábitos saudáveis. Ademais, parte da premissa que o indivíduo transita por estágios de motivação para a mudança de comportamento, a seguir: pré-contemplação, contemplação, ação e manutenção, que se aplicam tanto ao comportamento de uso quanto ao tratamento (Oliveira et al, 2003), sem esquecer que a recaída pode estar presente em algum momento.

No segundo momento, foi apresentado a temática a ser trabalhada relatando sobre os estágios de mudança. Os participantes se mostravam atentos durante toda a apresentação realizada pelos acadêmicos, fizeram perguntas, relataram exemplos de suas vivências e contribuíram de forma significativa para que o conteúdo programado fosse compreendido pela maioria.

Em seguida, utilizando-se um instrumento (Figura 1) contendo os nomes dos estágios de motivação, cada usuário foi convidado a participar para discutir em qual estágio se encontravam no momento, relatando o motivo e as características que possuíam pela escolha. Esse momento foi proveitoso e possibilitou que os participantes se sentissem à vontade para compartilhar suas experiências, dúvidas e anseios sobre o sentimento de motivação para o tratamento, bem como sobre as 
expectativas após saírem da Unidade de Acolhimento.

Os participantes reconheceram que no início do contato com a substância psicoativa se encontravam apenas na fase de pré-contemplação (antes da admissão na Unidade), que se refere ao estado que a pessoa não percebe a real dimensão dos problemas com a substância psicoativa e o estágio de contemplação que se caracteriza por considerar a possibilidade de mudar o comportamento e avaliar meios para a sua concretização (Oliveira et al, 2003).

Ao indicarem o estágio que se encontravam no momento do grupo operativo, a maioria dos participantes considerou estar no período de ação, pelo motivo de estarem buscando ajuda profissional e orientações em um equipamento de saúde da RAPS. Ademais, a maioria mencionou que também estava no estágio de manutenção, uma vez que se trata do período onde se trabalha a prevenção da recaída.

Figura 1. Material utilizado para identificação dos estágios de mudança. Fortaleza-CE, 2019.

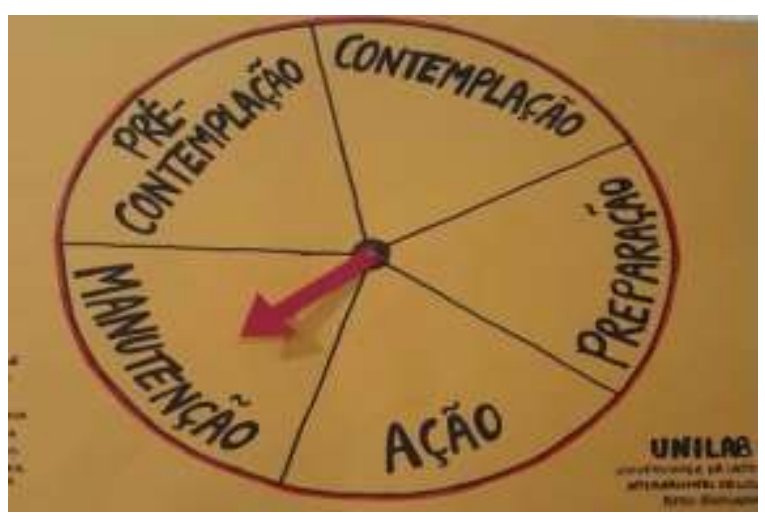

Fonte: Autores.

\subsection{Terceiro momento - Encerramento da atividade}

Para finalizar, o terceiro momento do grupo operativo foi marcado por emoção dos participantes e muitos se identificaram com as palavras mencionadas. Ademais, foi incentivado que cada participante citasse uma palavra retratando um sentimento positivo ou o que a atividade realizada representou para o seu processo de mudança. Assim, as principais palavras mencionadas foram: gratidão, amor, felicidade, sonhos, autoconfiança, respeito e vida.

Contudo, na hora de avaliar sobre a apresentação, os participantes mencionaram ainda as poucas atividades realizadas no cotidiano da unidade e a ociosidade, sem atividades de grupo e recreativas. Todavia, houve ainda uma situação peculiar onde dois usuários se desentenderam, e foi necessário mudá-los de lugar, para que só assim pudessem dar continuidade ao grupo. Assim, os resultados esperados eram que eles participassem de forma ativa da atividade, visto que já fazia um tempo que não tinha a realização de um grupo terapêutico na unidade.

O grupo operativo é um meio em que os usuários debatem sobre assuntos que sejam da necessidade de todos, em que ocorre a troca de experiências e a melhoria na adaptação no modo de vida coletivo (Nascimento \& Galindo, 2017). Estruturalmente, um grupo operativo é composto pelos integrantes, um coordenador e um observador. Além disso, é necessária a combinação, com os integrantes, de regras básicas de funcionamento, ou seja, de um enquadre grupal. Este, além de objetivos explícitos, tem como objetivos implícitos propiciar senso de limites e de realidade a cada integrante e ao grupo e, também, manter a coesão e dar continência às ansiedades emergentes no campo grupal, contribuindo, com isso, para que se cumpra sua função terapêutica.

As atividades grupais são de essencial importância enquanto práticas educativas para o desenvolvimento humano, ajudando as pessoas a relacionar-se melhor consigo mesma, com o outro e com o mundo (Mazzuchello et al, 2014). 
Acompanhar dependentes químicos, é um trabalho que exige conhecimento específico para lidar com as dificuldades e lutas diárias do grupo. O fortalecimento do cuidado de Enfermagem junto ao grupo operativo tem mostrado bons resultados, e a necessidade de um crescimento nessa área, a fim de beneficiar tanto os pacientes quanto os profissionais nos cuidados prestados.

O presente trabalho propôs uma reflexão acerca das questões que envolvem o cuidado em saúde mental e as práticas desempenhadas no âmbito dos serviços considerados substitutos, de acordo com os pressupostos da Reforma Psiquiátrica e a atual Política de Saúde Mental no Brasil. Ressalta-se, que aprendizagem centrada nos processos grupais coloca em evidência a possibilidade de uma nova elaboração de conhecimento. A aprendizagem é um processo contínuo em que a comunicação e interação são indissociáveis, na medida em que aprendemos a partir da relação com os outros.

O grupo operativo proporciona um momento de troca de conhecimentos, de interação dos próprios membros uns com os outros, pois entendem que apesar de ter passado por situações diferentes, naquele momento todos estavam em prol de um mesmo objetivo e são eles fonte de apoio muitas vezes para que os outros não desistam quando se tem o pensamento na recaída. Além disso, proporciona uma confiança maior nos profissionais, para que desta forma, identifiquem as reais necessidades para poder contribuir com o apoio, possam desenvolver uma escuta ativa e tenham uma relação melhor com os pacientes.

A motivação do dependente químico se destaca como um dos critérios primordiais para a procura e manutenção do tratamento (Janeiro, 2007) e esse estado de prontidão é fundamental para reverter o comportamento problema e superá-lo. Reconhecer o problema é o ponto-chave para a pessoa mudar e isso pode acontecer através de abordagens motivacionais (Ferrreira et al ,2015).

Frente as alterações comportamentais em relação às mudanças de hábitos de vida dos usuários de drogas no transcorrer do tratamento, compreender melhor as barreiras e dificuldades que eles enfrentam auxilia na elaboração de intervenções que considerem suas reais necessidades (Ferreira et al, 2016). Ademais, identificar de forma assertiva o estágio de motivação para a mudança também se torna fundamental, visto que possibilita ao profissional de saúde a utilização de estratégias certas de acordo com o momento que o indivíduo se encontra para alcançar melhores resultados.

A disposição do indivíduo para mudar sua relação com a droga permite uma melhor compreensão da sua dependência química que auxilia na concretização de ações de mudança de comportamento. Ademais, a voluntariedade no tratamento e a força de vontade são aspectos essenciais para que esse processo de mudança seja positivo e esteja presente em todas as fases de reabilitação (Almeida et al, 2018).

Assim, foi visto que em cada momento do que foi planejado, os participantes agiam com foco e que necessitavam urgentemente de motivação para superar a fase de abstinência da substância, pois relataram que não é um percurso fácil e que precisavam de atividades que fortalecesse o processo de se autoconhecer para prepará-los para o momento da alta e para que não desistam do tratamento.

A realização de um grupo operativo é algo que pode ser planejado, entretanto, certos momentos fogem do cronograma proposto e requer do coordenador do grupo preparo suficiente para agir nestas situações com conhecimentos específicos. Logo, exige dos profissionais além do conteúdo teórico sobre a temática, fazendo-se necessário o cotidiano da prática da formação de grupos para fins terapêuticos, compreendendo esse momento como um espaço de escuta.

Desta forma, com o presente estudo, pode-se perceber como é importante que o profissional que atuará com este público, tenha conhecimentos na área, com cursos e uma especialização, para contribuir de forma significativa no tratamento desses pacientes, de forma ativa com intervenções que contribuam com a qualidade de vida dos usuários.

Sendo assim, a técnica de grupo operativo consiste em um trabalho cujo objetivo é promover um processo de aprendizagem em equipe. Dessa forma, aprender em conjunto significa uma leitura crítica da realidade e uma abertura para 
novos aprendizados.

\section{Considerações Finais}

A realização desta atividade proporcionou a troca de conhecimentos mútuos, por meio dos relatos de vida e experiência dos usuários de substâncias psicoativas, que os diferenciam de tal forma, que retratam que a estratégia de enfrentamento de um usuário pode não ter nenhum efeito para o outro, pois vivem processos de vidas diferentes. Contudo, eles apresentam algo em comum que é o desejo de superação e força de vontade para conviver sem o uso das substâncias psicoativas. A motivação para eles é primordial, e deve ser trabalhada continuamente pela equipe multiprofissional, para que juntos possam enfrentar as adversidades no tratamento.

O grupo é um conjunto de pessoas reunidas com uma finalidade em comum, e é uma experiência rica de conhecimentos não só para os pacientes como para quem coordena a experiência em questão. Ademais, vale ressaltar a importância de um grupo operativo como um instrumento fundamental no processo de cuidar e que contribui para melhorar a qualidade da assistência.

Ressalta-se que a atividade grupal foi bastante interessante e enriquecedora para as acadêmicas, pois foi possível compreender melhor a dinâmica de funcionamento dos grupos terapêuticos em uma Unidade de Acolhimento. Além disso, propiciou o desenvolvimento de habilidades e competências para que estejam aptas a agirem com respeito, sensibilidade e empatia, visto que para preparar um grupo terapêutico requer criatividade e conhecimentos sobre a finalidade de um grupo e foge do modelo centrado apenas na doença, visto que pode ser abordado assuntos diversos.

Destaca-se como uma importante limitação do estudo a inviabilidade de dar continuidade ao grupo terapêutico com diversos temas em outros momentos, para que assim, obtivessem melhores resultados, facilitando a reabilitação dos usuários do serviço. Para tanto, preconizam-se mais estudos para abordar esta temática.

\section{Referências}

Almeida, R. B. F., Santos, N. T. V., Brito, A. M., Silva, K. S., \& Nappo S. A. (2018). O tratamento do dependente na perspectiva das pessoas que fazem uso de crack. Interface (Botucatu), 22(66), 745-756. https://www.scielo.br/pdf/icse/v22n66/1807-5762-icse-22-66-0745.pdf

Bastos, A. B., \& Bastos I. (2010) A técnica de grupos-operativos à luz de Pichon-Rivière e Henri Wallon. Psicólogo inFormação, 14(14) 160-169. http://pepsic.bvsalud.org/pdf/psicoinfo/v14n14/v14n14a10.pdf

Brasil. Ministério da Saúde. (2017). Secretaria de Atenção à Saúde, Departamento de Atenção à Saúde Mental. Rede de Atenção Psicossocial, e dá outras providências. https://bvsms.saude.gov.br/bvs/saudelegis/gm/2017/prt3588_22_12_2017.

Ferreira, A. C. Z., Capistrano, F.C., Souza, E.B., Borba, L. O., Kalinke, L. P., \& Maftum, M. A. (2015). Motivações de dependentes químicos para o tratamento: percepção de familiares. Rev. Bras. Enferm, 68 (3), 474-481. https://doi.org/10.1590/0034-7167.2015680314i

Ferreira, A. C. Z., Czarnobay, J., Borba, L. O., Capistrano, F.C., Kalinke, L. P., \& Maftum, M. A. (2016). Determinantes intra e interpessoais da recaída de dependentes químicos. Rev. Eletr. Enfermagem, 18. https://revistas.ufg.br/fen/article/view/34292

Inoue, L., Bellini, L. C., Paiano, M., Haddad, M. C. L., Marcon, S. S. (2019). Percepções de vida e perspectivas de futuro de usuários de drogas: compreender para cuidar. Rev Eletrônica Saúde Mental Álcool Drog, 15(2), 52-59. http://pepsic.bvsalud.org/pdf/smad/v15n2/08.pdf

Janeiro L. (2007). Motivação para o tratamento: estratégia psicoterapêutica no tratamento da toxicodependência. Rev Toxicodependência, 13 (1), 49-58. https://www.researchgate.net/publication/266007069

Mazzuchello, F. R., Ceretta, L. B., Schwalm, M. T., Dagostim, V. S., Soratto, M. T. (2014). A atuação dos enfermeiros nos grupos operativos terapêuticos na estratégia saúde da família. O Mundo da Saúde, 38 (4), 462-472. http://www.saocamilo-sp.br/pdf/mundo_saude/155566/A11.pdf

Moraes, L. M. P., Lopes, M. V. O., \& Braga, V. A. B. (2006). Componentes funcionais da teoria de Peplau e sua confluência com o referencial de grupo. Rev Acta paul. Enferm, 19 (2), 228-233.

Nascimento, T., \& Galindo, W. C. M. (2017). Grupo operativo em centros de atenção psicossocial na opinião de psicólogas. Rev Pesquisas e Práticas Psicossociais, 12 (2), 422-438. http://pepsic.bvsalud.org/pdf/ppp/v12n2/13.pdf

Silva, E. R., Ferreira, A. C. Z., Borba, L. O., Kalinke, L. P., Nimtz, M. P., \& Maftum, M. A. (2016). Impacto das drogas na saúde física e mental de dependentes químicos. Rev Ciencia Cuid Saude, 15(1), 101-108. 10.4025/cienccuidsaude.v15i1.27137 
Research, Society and Development, v. 10, n. 3, e43510313318, 2021

(CC BY 4.0) | ISSN 2525-3409 | DOI: http://dx.doi.org/10.33448/rsd-v10i3.13318

Soares, I., Esswein, G.C., \& Benetti, S. P. C. (2017). Motivação para mudança em homens e mulheres dependentes de crack. Rev. Psicologia, Saúde e Doenças, 18(2): 567-580. http://www.scielo.mec.pt/pdf/psd/v18n2/v18n2a23.pdf

Oliveira, D. M.., Rena, P. B. O., Mendonça, E. T., Pereira, E. T., Jesus, M. C. P., Merighi, M. A. B. (2016). O grupo operativo como instrumento de aprendizagem do cuidado por mães de filhos com deficiência. Escola Anna Nery, 20(3), e20160077. https://dx.doi.org/10.5935/1414-8145.20160077

Oliveira, M. S., Laranjeira, R., Araújo, R. B., Camilo, R. L., \& Schneider, D. D. (2003). Estudo dos estágios motivacionais em sujeitos adultos dependentes do álcool. Rev.Psicologia: Reflexão e Crítica, 16(2), 265-270. https://www.scielo.br/pdf/prc/v16n2/a06v16n2.pdf

Pinheiro, C. W., Araújo, M. A. M., Rolim, K. M. C., Oliveira, C. M., \& Alencar, A. B. (2019). Teoria das relações interpessoais: reflexões acerca da função terapêutica do enfermeiro em saúde mental. Rev. Enfermagem em Foco, 10(3), 64-69. http://revista.cofen.gov.br/index.php/enfermagem/article/view/2291/580

Tavares, C. M., \& Mesquita, L. M. (2019). Sistematização da assistência de enfermagem e clínica ampliada: desafios para o ensino de saúde mental. Rev. Enfermagem em Foco, 10(7), 121-127. http://revista.cofen.gov.br/index.php/enfermagem/article/view/2810 